\title{
My C.G.S.I.S.A.H. Theory of Dark Matter
}

\section{Eugene Terry Tatum}

Bowling Green, KY, USA

Email: ett@twc.com

How to cite this paper: Tatum, E.T. (2019) My C.G.S.I.S.A.H. Theory of Dark Matter. Journal of Modern Physics, 10, 881-887. https://doi.org/10.4236/jmp.2019.108058

Received: June 10, 2019

Accepted: July 2, 2019

Published: July 5, 2019

Copyright $\odot 2019$ by author(s) and Scientific Research Publishing Inc. This work is licensed under the Creative Commons Attribution International License (CC BY 4.0).

http://creativecommons.org/licenses/by/4.0/

\begin{abstract}
Theory and observations concerning the cosmic reionization epoch are briefly discussed in the context of recent observations attributed to dark matter. A case is made that cold ground state interstellar atomic hydrogen of average density of about one atom per cubic centimeter $\left(1.67 \times 10^{-21} \mathrm{~kg} \cdot \mathrm{m}^{-3}\right.$ or $1.67 \times$ $10^{-24} \mathrm{~g} \cdot \mathrm{cm}^{-3}$ ) appears to be the most likely candidate to explain these observations.
\end{abstract}

\section{Keywords}

Dark Matter, Early Universe, Reionization Epoch, Dark Ages, Cosmology

Observations, Galaxies: ISM, ISM: Atoms, Radio Lines: ISM

\section{Introduction and Background}

In May of 2019, I shared my C.G.S.I.S.A.H. theory of dark matter with colleagues at the dark matter workshop sponsored by the World Science Festival. What follows is a brief note concerning the new constraints on dark matter and a discussion of my conjecture and its observational predictions.

Convincing observational support for dark matter begins with the publication by Rubin and Ford [1] concerning unexpected galactic rotation curves. These observations, soon followed by others [2], provide strong support that an invisible (i.e., "dark") form of gravitationally attractive matter within the interstellar vacuum is contributing to galaxies approximately 5 - 10 times the total mass of the visible galactic matter (i.e., stars, warm molecular gas clouds, and dust). By "invisible" it is meant that this matter is not emitting any detectable light.

It has subsequently become apparent that one can further observe the effect of this dark matter by its gravitational lensing properties. By these observations, there appears to be a roughly spherical cloud (i.e., a "halo") of dark matter gas or superfluid extending up to approximately $200 \mathrm{kpc}$ from the observed galactic centers. Dark matter is also nearly collisionless due to a low scattering cross-section, 
as deduced from Tucker's observations of the bullet cluster [3] and other colliding galaxy clusters. Furthermore, the Planck Collaboration report [4] of the cosmic microwave background (CMB) anisotropy indicates that dark matter was present at the time of the recombination/decoupling epoch. It is even postulated that dark matter has been the seeding structural scaffolding for the further formation of galaxies, galaxy clusters and filaments in the subsequent evolution of the universe.

In recent years, numerous theories and detection methods have been proposed for dark matter with the above properties. While it is not within the scope of this paper to review the many publications on this subject, three important publications in 2018 and two important publications in 2019 deserve special mention herein.

The first of these is Barkana's review [5] of the reionization epoch ("cosmic dawn") 21-cm observations. These observations constrain dark matter to a very slow-moving (i.e., cold) particle with a mass-energy of no greater than 2 - 3 $\mathrm{GeV}$. Furthermore, the graph on page 9 of the Barkana reference shows a very tight correlation between a dark matter particle of about $0.938 \mathrm{GeV}$ and the minimum possible $21-\mathrm{cm}$ brightness temperature $T_{21}$ at redshift $z=17$. Thus, atomic hydrogen appears to be the only baryon not yet ruled out by these new tight constraints.

The second reference of importance in 2018 is Posti and Helmi's analysis [6] of Gaia data extracted from a $20 \mathrm{kpc}$ (65.2 thousand light-years) radius halo sphere centered at the Milky Way center. From their analysis one can deduce the ratio of dark matter to visible matter within this halo sphere to be approximately 1.37 to 0.54 , or 2.54 to one. This ratio will be further addressed in the Results section to follow.

The third reference of importance in 2018 is physicist Stacy McGaugh's publication entitled, "Strong Hydrogen Absorption at Cosmic Dawn: The Signature of a Baryonic Universe" [7]. One should carefully read the McGaugh reference for the reasoning that the cosmic dawn observations fit best for baryonic dark matter. Thus, nonbaryonic proposals for dark matter do not appear to be necessary.

The first reference of importance in 2019 is the Read publication [8] which provides support for "dark matter heating" within active galactic centers. This process may explain why active galactic centers tend to have a somewhat shallower dark matter core. Thus, dark matter heating may be an important variable in understanding its perplexing spatial distribution, particularly with respect to the dark matter "cusp-core problem."

The second reference of importance in 2019 is the March online report [9] of the Gaia-Hubble Collaboration. Here, for the first time, one can have confidence that the "visible matter mass" of the Milky Way is approximately 250 billion $\mathrm{M} \odot$. Therefore, if one can assume that this visible matter mass is roughly confined to within the $20 \mathrm{kpc}$ Posti and Helmi radius halo sphere, their 2.54 ratio would imply approximately 635 billion $\mathrm{M} \odot$ of dark matter within $20 \mathrm{kpc}$ of the 
Milky Way center.

With all of the above observations concerning dark matter, one can now construct the following table (Table 1) of these features with the relevant references listed in the right-hand column.

Given these features characteristic of dark matter, it is useful to review what observations suggest about the evolution of the universe since the recombination/decoupling epoch. During the adiabatic cooling period of the cosmic "dark ages" the positive feedback of gravitational attraction is thought to have accentuated the anisotropy we now observe in the cosmic microwave background (CMB) by creating centripetal movements of the atomic hydrogen within the denser regions of the CMB map. In contrast to these collapsing and swirling clouds of the nascent stars and galaxies, the intervening atomic hydrogen within the minimum density regions of the $\mathrm{CMB}$ map is thought to have been relatively motionless (i.e., colder). With the continuing cosmic expansion, this intervening atomic hydrogen, the primary matter in regions we now refer to as the intergalactic and interstellar vacuum, would have ultimately become so sparse as to be nearly collisionless and predominantly confined to the ground state (except where in close proximity to the nascent stars). At the beginning of the reionization epoch (i.e., "cosmic dawn") the Wouthuysen-Field effect of the Lyman-alpha radiation of the first stars should have reduced the spin temperature $T_{S}$ of ground state interstellar atomic hydrogen to well below the CMB radiation temperature $T_{R}[10]$. Such a temporary decoupling from the CMB radiation temperature would have eventually resolved due to the increasing stellar black body radiation closing out the cosmic dawn epoch.

Astrophysical observations of the $21-\mathrm{cm}$ absorption line in the redshift $z$ range corresponding (in standard cosmology) to approximately 110 - 250 million years after the Big Bang show evidence of a process very much like this, as seen in Figure 1 [11]. However, the conventional wisdom is that a mysterious nearly collisionless non-baryonic cold dark matter must have also been present in the interstellar vacuum, as a required intermediary in this process.

Table 1. Dark matter features and relevant references.

\begin{tabular}{|c|c|}
\hline Dark Matter Features & References \\
\hline Cold (i.e., low velocity) & Barkana \\
\hline No Emissions (i.e., dark) & Rubin \& Ford \\
\hline Collisionless (i.e., low cross-section) & Tucker \\
\hline Baryon Expected & McGaugh \\
\hline Mass-Energy less than $3 \mathrm{GeV}$ & Barkana \\
\hline Dark Matter $\mathrm{M}_{20 \mathrm{kpc}} 635$ Billion $\mathrm{M} \odot$ & Gaia/Posti \& Helmi \\
\hline Central DM Heating (“coring”) & Read \\
\hline CMB Decoupling at Dawn & Astrobaki/McGaugh \\
\hline Structural Scaffold & Planck \\
\hline Existence at CMB Emission & Planck \\
\hline
\end{tabular}




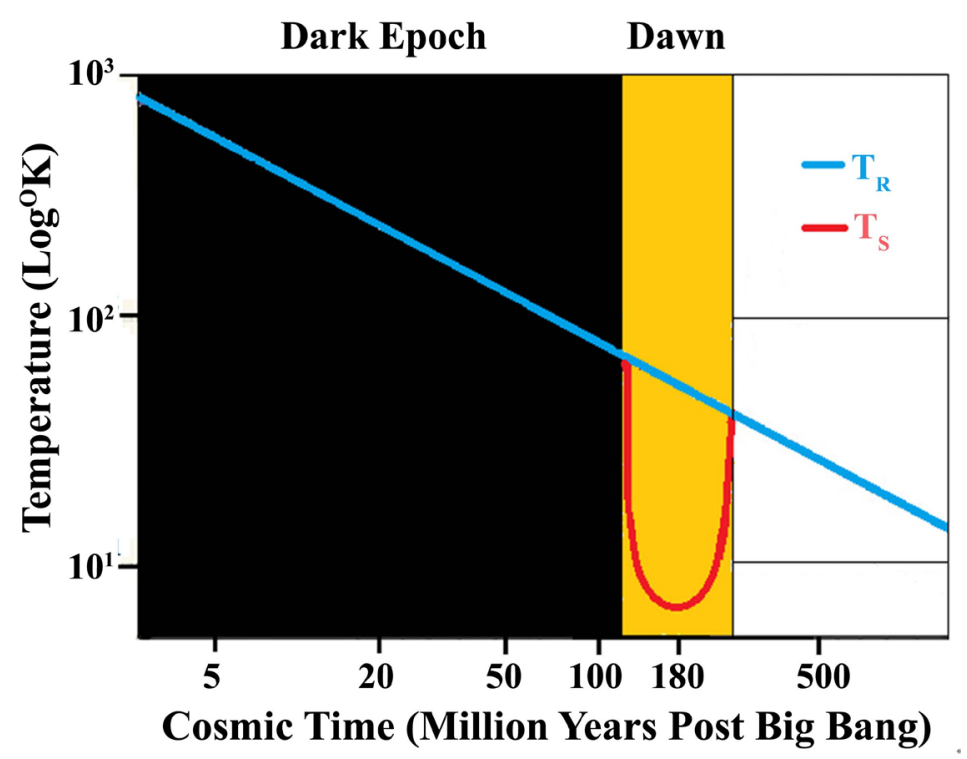

Figure 1. Radiation temperature $\left(T_{R}\right)$ and spin temperature $\left(T_{S}\right)$ vs time.

Unfortunately, the reasoning that such an intermediary nonbaryonic matter was required for this process is also somewhat mysterious, because a temporary decoupling from the $\mathrm{CMB}$ radiation temperature is to be expected in a purely baryonic universe (see McGaugh [7]).

Perhaps the ongoing search for exotic dark matter also reflects a misunderstanding about the current abundance of cold ground state interstellar atomic hydrogen in comparison to the constituents of the visible stars, warm molecular gas clouds and dust in our galaxy. It should be remembered that ground state interstellar atomic hydrogen coupled to the $\mathrm{CMB}$ radiation temperature (as was also undoubtedly present in great abundance during the "dark ages") is essentially invisible to modern detectors, except where its characteristic $21-\mathrm{cm}$ absorption line is "backlit" by distant starlight.

The Milky Way disc rotates with a period of approximately 250 million years [12]. Based upon the Baryonic Tully-Fisher relation [13] and the March 2019 Gaia-Hubble Collaboration report, the sum total mass of the visible stars, warm molecular gas clouds and dust in the Milky Way is reliably estimated to be 250 billion $\mathrm{M} \odot$. And yet, the calculated amount of ground state interstellar atomic hydrogen coupled to the $\mathrm{CMB}$ radiation temperature within a $20 \mathrm{kpc}$ halo radius of the Milky Way center actually dwarfs this total visible matter mass estimate (see calculation below). This reflects the vastness of the interstellar vacuum in comparison to the visible matter.

\section{Results}

Line-of-sight measurements of the hyperfine $21-\mathrm{cm}$ absorption line (within the light from distant stars of a known distance from the observer) allow one to estimate an average density of cold ground state interstellar atomic hydrogen of approximately one atom $/ \mathrm{cm}^{3}$ (or $1.67 \times 10^{-21} \mathrm{~kg} \cdot \mathrm{m}^{-3}$ ) [14] [15] [16]. 
One can now use this knowledge in the context of Posti and Helmi's recent Gaia survey analysis of the Milky Way (see reference [6]). They report the ratio of dark matter to visible matter within a $20 \mathrm{kpc}$ spherical halo radius of the galactic center to be approximately 1.37 to 0.54 . This simplifies to a ratio of approximately 2.54. If we assume the above current best estimate of the Milky Way visible matter mass ( 250 billion $\mathrm{M} \odot$ is equal to $4.97 \times 10^{41} \mathrm{~kg}$ ) and divide that by the volume of a galactic halo sphere of $20 \mathrm{kpc}$ radius $\left(9.85 \times 10^{62} \mathrm{~m}^{3}\right)$, the average visible matter density within that galactic halo sphere is $5.05 \times 10^{-22} \mathrm{~kg} \cdot \mathrm{m}^{-3}$, approximately one-third of the above-mentioned average density of cold ground state interstellar atomic hydrogen! Multiplying $1.67 \times 10^{-21} \mathrm{~kg} \cdot \mathrm{m}^{-3}$ by a 0.83 correction factor (for the expected slightly lower ground state atomic hydrogen density in the halo sphere portion outside the galactic disk) times the $20 \mathrm{kpc}$ radius galactic halo sphere volume gives an estimated mass of cold ground state interstellar atomic hydrogen of $1.37 \times 10^{42} \mathrm{~kg}$, in other words approximately 689 billion $\mathrm{M} \odot$, within that sphere. The corresponding 2.76 ratio (from dividing 689 billion $\mathrm{M} \odot$ by 250 billion $\mathrm{M} \odot$ ) is well within the margin of error of Posti and Helmi's observed ratio of dark matter to visible matter for the same $20 \mathrm{kpc}$ radius galactic halo sphere.

\section{Discussion}

The calculations made in the Results section suggest the strong possibility that cold ground state interstellar atomic hydrogen averaging approximately one atom $/ \mathrm{cm}^{3}$ is what we currently refer to as cold dark matter (CDM). The following table (Table 2) compares the above-mentioned dark matter features with sparse interstellar atomic hydrogen coupled to the $\mathrm{CMB}$ temperature.

The origin of the C.G.S.I.S.A.H. acronym becomes apparent by reading down the letters in the right-hand column, which are abbreviations for the top five rows of the table. The abbreviation W-F effect stands for the Wouthuysen-Field effect on ground state neutral atomic hydrogen due to Lyman-alpha radiation

Table 2. Dark matter features vs. interstellar $\mathrm{H}$ features.

\begin{tabular}{|c|c|c|}
\hline Dark Matter Features & Interstellar $\mathrm{H}$ at 1 atom $/ \mathrm{cm}^{3}$ & CDM \\
\hline Cold (i.e., low velocity) & CMB Equilibrated & $\mathrm{C}$ \\
\hline No Emissions (i.e., dark) & Ground State & GS \\
\hline Collisionless (i.e., low cross-section) & Interstellar/Sparse & IS \\
\hline Baryon Expected & Atomic Hydrogen & A \\
\hline Mass-Energy less than $3 \mathrm{GeV}$ & $0.938 \mathrm{GeV}$ Neutral H & $\mathrm{H}$ \\
\hline Dark Matter $\mathrm{M}_{20 \mathrm{kpc}} 635$ Billion M९ & 689 Billion $\mathrm{M} \odot$ & \\
\hline Central DM Heating (“coring”) & Loses Ground State & \\
\hline CMB Decoupling at Dawn & W-F Effect & \\
\hline Structural Scaffold & Most Abundant Atom & \\
\hline Existence at CMB Emission & Most Abundant Atom & \\
\hline
\end{tabular}


beginning with the first starlight of cosmic dawn. There is a nice discussion of this temporary $\mathrm{CMB}$ decoupling phenomenon in the AstroBaki reference. According to this reference, "...the W-F effect remains the dominant effect until reionization is complete." Once reionization was complete, the interstellar atomic hydrogen presumably became once again coupled to the CMB temperature, which is assumed to be the case at present.

As for future observable consequences of my dark matter conjecture presented herein, one can point to the ongoing refinement of observational constraints on the mass-energy of the dark matter particle. The studies to date appear to eliminate any baryonic particle much greater than about $1 \mathrm{GeV}$ (see Barkana [5]). However, they do not yet exclude neutral atomic hydrogen, with its mass-energy of $0.938 \mathrm{GeV}$. I predict that these constraints will further tighten around a dark matter particle with a mass-energy of $0.938 \mathrm{GeV}$. Furthermore, the sophisticated dark matter/baryon interaction simulations being conducted at the Kavli Institute for Particle Astrophysics and Cosmology have not yet simulated the dark matter candidate in these interactions as cold ground state interstellar atomic hydrogen of average density of about $1.67 \times 10^{-21} \mathrm{~kg} \cdot \mathrm{m}^{-3}(1.67 \times$ $\left.10^{-24} \mathrm{~g} \cdot \mathrm{cm}^{-3}\right)$ (R. Wechsler, Director, per verbal communication with this author on March 30, 2019). It is my prediction that such simulations will correlate nicely with dark matter observations, even to the extent of simulating central galactic coring (i.e., relative dark matter depletion) due to "dark matter heating" within active galactic centers (see Read [8]). Thus, the previously unexplained galactic and peri-galactic dark matter spatial distribution may be best understood in terms of heating and other dynamic effects upon the distribution of cold ground state interstellar atomic hydrogen.

\section{Conclusion}

For the above theoretical and observational considerations, the distinct possibility that the dark matter candidate could ultimately prove to be the ubiquitous but incredibly sparse (and thus nearly collisionless) cold ground state interstellar atomic hydrogen must be seriously entertained.

\section{Conflicts of Interest}

The author declares no conflicts of interest regarding the publication of this paper.

\section{References}

[1] Rubin, V. and Ford, W.K. (1970) The Astrophysical Journal, 159, 379. https://doi.org/10.1086/150317

[2] Rubin, V., et al. (1985) The Astrophysical Journal, 289, 81. https://doi.org/10.1086/162866

[3] Tucker, W. (2006) AIP Conference Proceedings, 801, 21-30.

[4] Aghanim, N., et al. (2018) Planck 2018 Results VI. Cosmological Parameters. 
arXiv:1807.06209v1

[5] Barkana, R. (2018) Cosmic Dawn as a Dark Matter Detector.

https://doi.org/10.1038/nature25791

[6] Posti, L. and Helmi, A. (2018) Astronomy \& Astrophysics, 621, A56. https://doi.org/10.1051/0004-6361/201833355

[7] McGaugh, S.S. (2018) Research Notes of the AAS, 2, 37. https://doi.org/10.3847/2515-5172/aab497

[8] Read, J.I., et al. (2019) Monthly Notices of the Royal Astronomical Society, 484, 1401-1420. https://doi.org/10.1093/mnras/sty3404

[9] Gaia-Hubble Collaboration (2019).

http://sci.esa.int/hubble/61198-hubble-and-gaia-accurately-weigh-the-milky-way-h eic1905

[10] AstroBaki (2017).

https://casper.ssl.berkeley.edu/astrobaki/index.php/Wouthuysen_Field_effect

[11] Bowman, J.D. (2018) Nature, 555, 67. https://doi.org/10.1038/nature25792

[12] Morris, M. (2002) The Milky Way. The World Book Encyclopedia, 551.

[13] Torres-Flores, S., et al. (2011) Monthly Notices of the Royal Astronomical Society, 416, 1936-1948. https://doi.org/10.1111/j.1365-2966.2011.19169.x

[14] Pananides, N.A. and Thomas, A. (1979) Introduction to Astronomy. Second Edition, Wiley \& Sons, New York, 293.

[15] Chaisson, E. and McMillan, S. (1993) Astronomy Today. Prentice Hall, New York, 418.

[16] Mammana, D.L. (2000) Interstellar Space. Popular Science, New York, 220. 